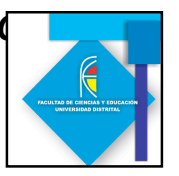

\title{
DESARROLLO DE HABILIDADES DE PENSAMIENTO CIENTÍFICO: UNA ESTRATEGIA DIDÁCTICA PARA NIÑOS DE LA ZONA ALTOS DE CAZUCÁ CON EL PROPÓSITO DE APROXIMARLOS A LA NOCIÓN DE TEMPERATURA.
}

\section{DEVELOPMENT OF SCIENTIFIC THINKING: A DIDACTICAL STRATEGY FOR CHILDREN OF ALTOS DE CAZUCÁ AIMED TO INTRODUCE THEM TO THE TEMPERATURE NOTION.}

\author{
Stefanny Jahel Quijano Urrego ${ }^{1}$ \\ Rusby Yalile Malagón ${ }^{2}$ \\ Germán Bautista ${ }^{3}$
}

\begin{abstract}
Resumen
La presente propuesta investigativa nace del trabajo de campo realizado en la Corporación Social Fe y Esperanza ubicada en Cazucá en el Barrio el Progreso. Esta propuesta ha permitido emprender una reflexión sobre la difícil situación que viven los niños por causa del desplazamiento y las condiciones de pobreza de su entorno. La intención es llevar a este escenario educativo una estrategia didáctica que favorezca el desarrollo de habilidades de pensamiento científico aproximándolos a la noción de temperatura. La propuesta investigativa favorece la recreación de espacios donde los niños desarrollen la capacidad de hacer preguntas, observar eventos, realizar descripciones sencillas y explicar algunos fenómenos naturales de su entorno; así mismo, es una opción para visualizar y diseñar nuevas alternativas para llevar la ciencia a los niños de estos contextos, estimulando su gusto por curiosear e indagar sobre lo que a su alrededor ocurre, explotando al máximo los espacios que para su estimulación ofrece el lugar donde habitan.
\end{abstract}

Palabras clave: Desplazamiento, habilidades de pensamiento, estado térmico.

\footnotetext{
${ }^{1}$ Estudiante de licenciatura en física universidad Pedagógica Nacional' stefannyquijnao@hotmail.com

${ }^{2}$ Docente Universidad Pedagógica Nacional

3 , Docente Universidad Pedagógica Nacional
} 


\section{Abstract}

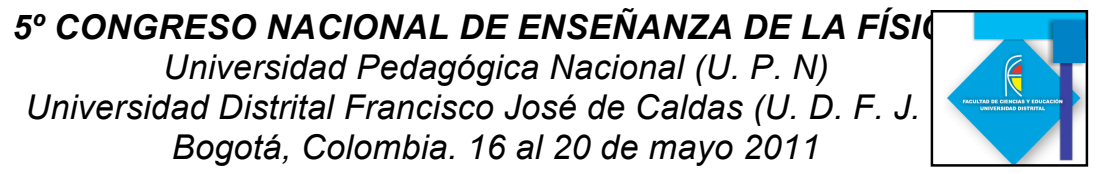

This work evolves from our intervention at the institution "Corporación Social Fé y Esperanza" at Bogota, Colombia. This work has been an opportunity to reflect on the harsh context the children has to endure due to their condition of being displaced from their home land. We aim to put forward a didactical strategy to improve the development of scientific thinking's skills by approaching them to de concept of temperature. In this strategy we set up contexts in which the students develop their ability to make question, to observe events, to realize description and to explain some natural phenomena of their environment. It is an option to see an design new ways to present science to children belonging to this contexts, by stimulating their taste to inquire and research on what happen in their surroundings, as well.

Keywords: Displacement, Scientific thinking's skills, Thermal state

\section{Introducción}

El presente escrito tiene la finalidad mostrar una propuesta de investigación realizada con los estudiantes de la Corporación Social Fe y Esperanza. La intención, fue llevar a este escenario educativo una estrategia didáctica para la enseñanza de las ciencias de grado tercero: buscando promover en ellos habilidades de pensamiento asociadas a su capacidad para hacer preguntas, observar eventos, realizar descripciones sencillas y expresar reflexiones sobre algunos fenómenos térmicos. El documento presenta algunas ideas generales sobre la población objeto de estudio, describe la estrategia didáctica diseñada y algunas ideas centrales asociadas a los hallazgos encontrados en la implementación.

\section{Sobre la Población}

La línea de profundización del departamento de física," Construcción del conocimiento científico desde la perspectiva de los enfoques didácticos" de la Universidad Pedagógica Nacional realiza observaciones e intervenciones didácticas en distintos colegios de municipio de Bogotá y sus alrededores con el fin de colaborar en la solución de las necesidades que estos presenten asociadas al proceso de enseñanza de las ciencias.

Uno de estos trabajos fue el realizado en la práctica pedagógica en la "Corporación Social Fe y Esperanza" el cual permitió reconocer y reflexionar sobre la difícil situación que los niños viven por causa del desplazamiento y las condiciones de pobreza en su entorno. Por esta razón se emprendieron reflexiones que permitieron reconocer en detalle algunas características de la población en términos cognitivos y sociales. Así mismo, permitió emprender acciones didácticas que estimularon el desarrollo de habilidades de pensamiento en el aprendizaje de las ciencias y generaron en los estudiantes una mirada diferente frente a los eventos naturales que los rodean. Los niños objeto de estudio tienen edades entre seis y ocho años y cursan grado tercero.

\section{Reflexiones Conceptuales}

Para desarrollar esta propuesta didáctica inicialmente se intentó realizar un reconocimiento sobre el concepto de desplazamiento su historia y las perspectivas desde 


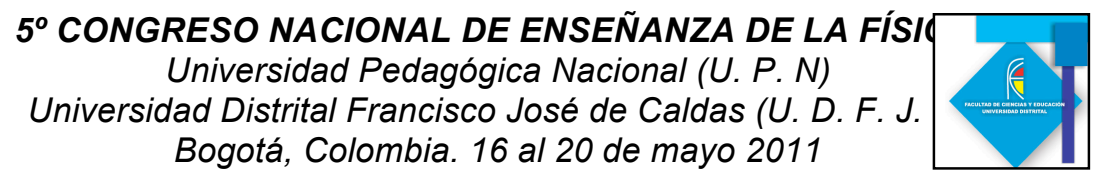

Las cuales se ha estudiado en nuestro país, debido a que Cazucá ha sido considerado un sector habitado por grupos humanos desplazados. Así mismo, se abordan algunas ideas de la pedagogía de Paulo Freire cómo un autor que logró comprender las condiciones de inequidad presentes en estas poblaciones.

Así mismo, se estudió el concepto de temperatura, como eje disciplinar que se pretendía abordar con los estudiantes. Esta revisión se realizó con la pretención de profundizar en el concepto y sus implicaciones sobre el diseño didáctico. Se asume que el lenguaje es una fuente muy importante de dificultades en la enseñanza de las ciencias, puesto que, las palabras en general tiene diversos significados en los diferentes contextos culturales, y se interiorizan en la cognición del individuo como resultado del signficado que su grupo social ha construido para el. Se trata entonces de establecer una conexión entre el simbolo y su significado,en nuestro caso el simbolo es "cualidad térmica" y el significado es aquello que el niño siente cuando habla de "frio o caliente". Se asume que el simbolo estado termico es el nombre que se le da a una cualidad de un cuerpo para explicar su comportamiento, es decir, la forma en la que interactua con el sujeto. El sujeto puede organizar todos los cuerpos de acuerdo a su estado térmico y hacer corresponder cada estado térmico con un número construyendo de esta manera una "escala de temperatura".

\begin{tabular}{|c|c|c|}
\hline $\begin{array}{l}\text { Intervención } 1 \\
\text { Diagnostico }\end{array}$ & $\begin{array}{l}\text { Intervención } \mathbf{2} \\
\text { Asignar un símbolo a la sensación } \\
\text { "frio y caliente" }\end{array}$ & $\begin{array}{l}\text { Intervención } \mathbf{3} \\
\text { Un instrumento más } \\
\text { preciso que mi tacto }\end{array}$ \\
\hline $\begin{array}{l}\text { Momento 1: Realizar } \\
\text { una priemera } \\
\text { observación y } \\
\text { descripción de un } \\
\text { objeto previamente } \\
\text { establecido,que } \\
\text { permita identificar } \\
\text { como se encuentran } \\
\text { las habilidades de los } \\
\text { niños para observar y } \\
\text { describir objetos de su } \\
\text { entorno. }\end{array}$ & $\begin{array}{l}\text { Momento } 1 \text { Relacionar la sensación } \\
\text { frío y caliente con el simbolo } \\
\text { "Estado Térmico" del sistema. } \\
\text { Actividades } \\
\text { Entrada: Reconociendo nuestros } \\
\text { compañeros con el tacto. } \\
\text { Experimentación: La arenita } \\
\text { Cierre:Dibujemos lo aprendido y } \\
\text { describamolo. } \\
\text { Momento } 2 \text { Clasificar y ordenar } \\
\text { según el estado térmico del sistema } \\
\text { Actividades } \\
\text { Entrada: Juego Perceptivo visual, } \\
\text { auditivo, u olfativo } \\
\text { Experimentación: Los vasitos de } \\
\text { agua. } \\
\text { Cierre: Dibujemos lo prendido y } \\
\text { describamolo. }\end{array}$ & 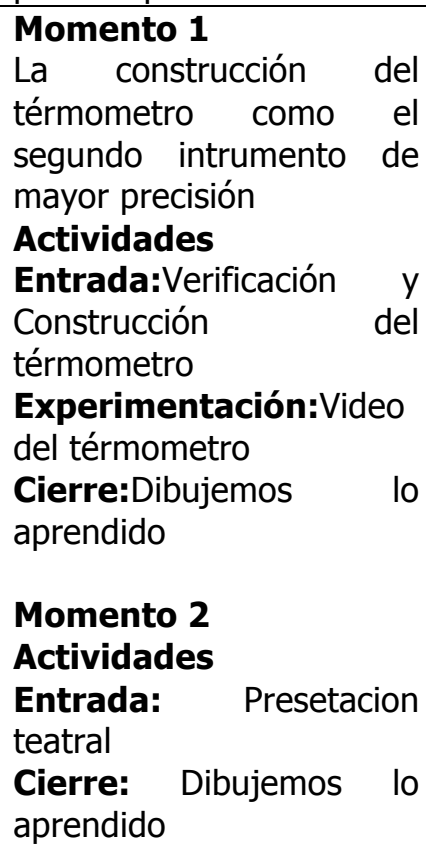 \\
\hline
\end{tabular}

Tabla 1.Esquema de la Estrategía Didáctica 


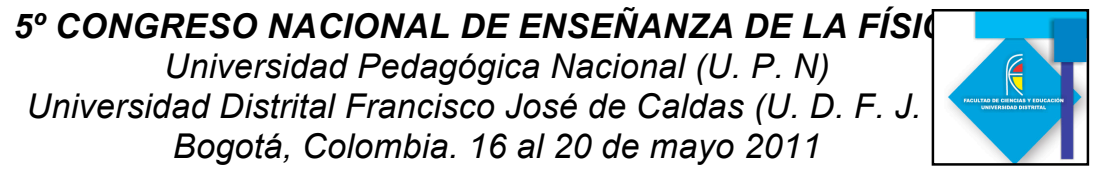

\section{Diseño y propuesta en marcha}

El diseño didáctico se enfatizó en los siguientes ejes: El papel del experimento en el aprendizaje de la física, las habilidades de pensamiento en el aprendizaje de las ciencias y el uso de imágenes y videos en el aprendizaje de los niños. La estrategía diseñada

consta de tres intervenciones. En cada intervención es posible encontrar uno o dos momentos, que corresponden a los momentos de las clases que se desarrollaron. Cada clase o momento tiene una actividad de entrada, una actividad de experimentación y una de cierre. El tiempo total de intervención es de 10 horas y el tiempo de cada momento o clase es de una hora y treinta minutos.

Para la primera intervención se tomaron como objetos a observar los seres vivos que rodeaban el entorno del niño, esto apropósito del gusto que los estudiantes tienen por los animales. El objetivo, para el docente, consisitía en identificar que cosas hacían los niños para observar y describir eventos o elementos de su entorno. En la intervención dos se trataba de llevar al estudiantes a asociar el término "cualidad térmica" con un conjunto de sensaciones y llevarlo a realizar una clasificación de los cuerpos según su estado térmico.En la tercera intervención se lleva a los estudiantes a reflexionar sobre las limitaciones que presenta el sentido del tacto al momento de precisar que tempertura tiene un cuerpo, la importancia de esta sesión radicó en la introducción de un instrumento de medida, mas sensible y preciso que el tacto y se dio inicio a la construcción del un térmometro.

\section{Intervención 1: Detalles del diagnóstico}

Momento 1: Exploremos los animales de nuestro entorno: Se reunirán en parejas y saldrán a explorar la zona, cada pareja de estudiantes se dirigirá al espacio establecido, para observar un ser vivo. Dentro de la actividad la maestra preguntará a cada pareja de estudiantes ¿Cual fue el ser vivo que eligieron? ¿Qué color tiene?, ¿Qué forma tiene?, ¿Se arrastra?, ¿Cuántas patas tiene?, para identificar la forma en la que los estudiantes observan y las estrategias que utilizan para describir los objetos observados.

Nota: Es posible que el maestro encuentre que los estudiantes realizaran sus descripciones de forma superficial y poco detallada, por lo tanto, debe acompañar de preguntas las descripciones y encaminar sus esfuerzos posteriores a estimular en los estudiantes la habilidad para observar en detalle los objetos del mundo natural y para tratar de describirlos con mayor fidelidad.

\section{Intervención 2: Asignar un símbolo a la sensación "frio y caliente"}

Momento 1: Relacionar la sensación frio y caliente con el símbolo "Estado Térmico" del sistema: En la actividad de entrada se les propone a los estudiantes hacer dos grupos. El primer grupo se forma en círculo, el segundo grupo forma un círculo dentro del círculo formado por el primer grupo. El segundo grupo tiene los ojos vendados, cada niño del círculo exterior debe reconocer a su compañero del círculo interior, tocando su cara con las manos. Cuando finalice la exploración, los del círculo externo se ubican en una hilera y los que tenían los ojos vendados se descubren los ojos para buscar a su compañero. Gana quien adivine quien era el 


\section{$5^{\circ}$ CONGRESO NACIONAL DE ENSEÑANZA DE LA FÍSI \\ Universidad Pedagógica Nacional (U. P. N) \\ Universidad Distrital Francisco José de Caldas (U. D. F. J. \\ Bogotá, Colombia. 16 al 20 de mayo 2011}

compañero. Se preguntará a cada estudiante, cómo pudo reconocer a su compañero, que estrategia utilizo para reconocerlo y adicionalmente sobre la importancia del sentido del tacto. En la siguiente actividad titulada la arenita, los estudiantes se reúnen en grupos de cuatro, a cada grupo se le presenta cuatro vasos llenos de arena a diferente temperatura, cada estudiante deberá experimentar la sensación que se siente al tocar cada vasito de arena para luego organizarlo de acuerdo a su sensación. Es probable que algunos niños organicen los vasos conformando una fila,

mientras que otros organicen los vasos de otra manera. En este momento es importante hacer una serie de preguntas para descubrir cuál fue el criterio que el estudiante uso para organizar los vasos. El maestro debe utilizar la expresión "estado térmico" para indicar a los niños que la organización que realizaron fue de acuerdo a este criterio, con el propósito de que los niños se aproximen al vocabulario y lo empiecen a utilizar en el transcurso de la experiencia. Se espera que los estudiantes hayan realizado la organización de frio a caliente o de caliente a frio, en el caso que haya estudiantes que no organicen los vasos según este criterio, el docente debe orientarlos para que todos lleguen a esta conclusión. Parara finalizar el maestro pedirá a cada estudiante que dibuje el orden que le dio a los vasos a y el color que le dan a cada vasito de arena de acuerdo a su sensación.

Momento 2: Clasificar y ordenar según el estado térmico del sistema.La Primera actividad consiste en hacer un círculo con los integrantes del grupo donde se rotara una serie de sensaciones, como auditivas, olfativas y táctiles posteriormente se eligen tres estudiantes donde cada uno organizará las sensaciones que sintieron y las clasificara como: Auditivas, olfativas y táctiles. La siguiente actividad se harán cuatro grupos de trabajo, a cada grupo se le asignara un conjunto de vasos a distintas temperaturas los cuales están en desorden sobre las mesas de trabajo, cada grupo de trabajo debe conformar grupos de vasos de acuerdo a sus sensaciones táctiles, el maestro debe llevar a los estudiantes a reflexionar sobre el criterio de clasificación que están utilizando para armar los grupos, posteriormente se pide a los estudiantes que armen grupos de acuerdo a su estado térmico, una vez que estén organizados se les pide que le asignen un número a cada grupo de vasos con agua, se espera que los estudiantes asignen los números de frio a caliente, siendo frio el número menor y caliente el número mayor, si esto no ocurre el maestro debe llevar a los chicos a esta reflexión. Al finalizar de manera individual los estudiantes deben dibujar la experiencia y de igual forma ponerle un color a la sensación que sintieron y describir la situación, para ver como interpretaron la actividad y si comprendieron de manera adecuada.

\section{Intervención 3: Instrumento más preciso que mi tacto}

Momento 1: La construcción del térmometro como el segundo intrumento de mayor precisión. En nuestra primera actividad cada estudiante tendrá vasos de agua a una temperatura que con el tacto no le sea posible encontrar diferencias. Se introduce la inquietud asociada al qué hacer cuando el tacto no puede hallar diferencias, de este modo se procede a la construcción de un instrumentó más preciso. Se socializa un video sobre el termómetro, el cual describe que es el termómetro, y cómo funcionan. Para finalizar el estudiante dibujara el montaje y describirá la practica realizada. Se hace énfasis en la observación y en la descripción detallada del instrumento. 


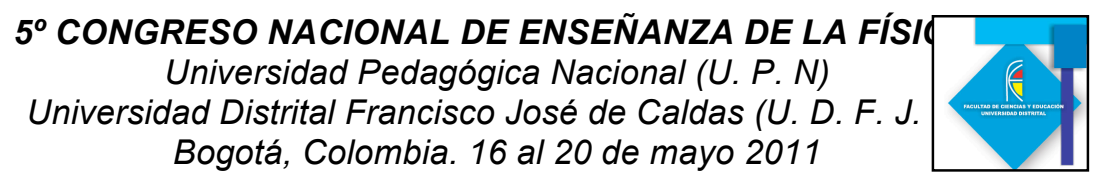

Momento 2: Se inicia con una presentación teatral a cargo del maestro, con el proposito de hacer un dialogo entre los personajes y los niños para intentar hacer visible la noción que los estudiantes lograron construir a través de la vivencia de la estrategia y visualizar en su vocabulario nuevas palabras asociadas a la temática.

\section{Conclusiones}

El diseño de estrategias didácticas para cualquier nivel de aprendizaje, pero en especial para la básica, demanda un fuerte trabajo conceptual desde la disciplina, pues le implica al maestro alcanzar comprensiones que le permitan construir puentes entre el lenguaje de la ciencia y lenguaje de los niños.

Es importante que los maestros que trabajan con poblaciones en condición de vulnerabilidad reflexionen sobre la importancia de llevar al aula estrategias y actividades que favorezcan la construcción de condiciones de equidad y de igualdad de oportunidades frente al conocimiento científico.

\section{Referencias Bliográficas.}

Zambrano, A. C. (2009). Historia y Epistemilogía en los Conceptos Básicos de la Termodinámica:Calor,temperatura y trabajo. Cali: Editorial Universidad del Valle. 